\title{
Seeing, Sensing and Recognizing Laban Movement Qualities
}

\author{
Sarah Fdili Alaoui* ${ }^{*}$ Jules Francoise ${ }^{\dagger}$, Thecla Schiphorst ${ }^{\dagger}$, Karen Studd ${ }^{\ddagger}$, Frederic Bevilacqua ${ }^{\mp}$ \\ * Univ. Paris-Sud, CNRS, Inria,Universit Paris-Saclay, Orsay, France. \\ $\dagger$ SIAT, Simon Fraser University, Surrey, BC, Canada. \\ Laban Institute of Movement Studies, NYC, USA. \\ ₹ STMS Lab, IRCAM-CNRS-UPMC, Paris, France. \\ saralaoui@lri.fr,jfrancoi@sfu.ca, thecla@sfu.ca, krnstudd@gmail.com, frederic.bevilacqua@ircam.fr
}

\begin{abstract}
Human movement has historically been approached as a functional component of interaction within human computer interaction. Yet movement is not only functional, it is also highly expressive. In our research, we explore how movement expertise as articulated in Laban Movement Analysis (LMA) can contribute to the design of computational models of movement's expressive qualities as defined in the framework of Laban Efforts. We include experts in LMA in our design process, in order to select a set of suitable multimodal sensors as well as to compute features that closely correlate to the definitions of Efforts in LMA. Evaluation of our model shows that multimodal data combining positional, dynamic and physiological information allows for a better characterization of Laban Efforts. We conclude with implications for design that illustrate how our methodology and our approach to multimodal capture and recognition of Effort qualities can be integrated to design interactive applications.
\end{abstract}

\section{ACM Classification Keywords}

I.5.2 Pattern Recognition: Design Methodology: Classifier design and evaluation

\section{Author Keywords}

Movement Qualities; Movement-based Interaction; Multimodal Measures; Movement Observation; Movement Recognition; Laban Movement Analysis.

\section{INTRODUCTION}

Computer Science have been inspired by recent developments within neuroscience and philosophy that showed the primacy of movement function and expression to the formation of our cognitive abilities [33]. However, within Human-Computer Interaction (HCI), human movement has historically been approached as a functional component of interaction. Early HCI research reflected a task-oriented focus and was preoccupied

(c) ACM 2015. This is the authorÕs version of the work. It is posted here by permission of ACM for your personal use. Not for redistribution. The definitive version is published in CHI 2017, May 06-11, 2017, Denver, CO, USA. with usability, ergonomics and efficiency. Recently, HCI has witnessed a shift in focus towards issues such as meaning, complexity, culture, emotion, lived experiences, engagement, motivation, and experience [10]. Movement gradually began to be considered not only for it functional properties, but also for its expressive and experiential values. Our work embraces this philosophy and argues for considering movement expertise to support more richly articulated human movement experience and expression within embodied interactions [9]. One missing, yet essential piece of the puzzle is the computational modeling of movement expressivity conveyed through movement qualities. This is an important question and a challenging computational task that remains unsolved. Although movement qualities are widely embodied and mastered in dance, performance or somatic practices, they are investigated by a marginal number of HCI researchers and remain the target of misinterpretations. In dance, movement qualities are defined as the manner in which movement is executed. They relate to the qualitative characteristics made manifest in movement.

Movement theories and systems have emerged in domains such as non-verbal communication, sign language and motor control. However, none of these systems articulates movement expressive qualities. One of the most notoriously used and investigated system that clearly formalizes movement qualities is that developed from the work of Rudolf Laban, called Laban Movement Analysis (LMA) [22]. It is both a somatic and embodied practice as well as an observational and analytical system. LMA historically emerged from dance but has been applied to various other contexts including HCI [29]. LMA conveys precise knowledge for the study of movement qualities formalized in the Effort theory.

The goal of our research is to develop a methodology for the computational modeling of movement qualities applied to interactive systems for dance. We argue that the computational modelling of Effort qualities should be informed by the expertise of Certified (Laban) Movement Analysts (CMAs) and particularly the visual or kinesthetic cues that they use to observe Effort qualities.

In this paper we first review the existing computational approaches to modeling movement qualities, and particularly those relying on Laban Effort theory. We then propose tree main contributions: (1) the integration of LMA expertise into 
the computational model of Efforts by interviewing CMAs on their movement observation; (2) a methodology for modeling Efforts based on a set of multimodal sensing and features that we designed while remaining faithful to the process of Effort observation; (3) a machine learning approach to the realtime continuous recognition of Effort qualities. We conclude this paper with implications for design that illustrate how our approach to multimodal capture and recognition of Effort qualities can be integrated to designing interactive applications.

While our applications focus on interactive systems for dance, our methodology and computational system for Efforts recognition can apply to other interaction contexts dealing with human movement and expression. Efforts represent "how" movement is performed, and are therefore present in all human movement. Our multimodal sensing methods and our recognition software can be adapted to integrate movement qualities into any movement based interactive system and thus can benefit broader HCI applications such as health, gaming, the quantitative self, and entertainment.

\section{EFFORT THEORY REVIEW}

LMA has a rich history. While originating from Rudolf Laban, a movement theorist and dancer [22], its contemporary applications include interdisciplinary fields such as HCI [29, $3]$, robotics [23, 31, 28] and cognitive science and psychology [25]. LMA is well situated to bridge movement theory, experience and movement knowledge representation across multiple domains.

The LMA system provides rigorous models for the description of movement, its function and its expression through four components defined as Body, Effort, Space and Shape (BESS). Body represents what is moving. Effort represents how the body is moving. Space represents where the body is moving. Shape represents the relationship of the body shape changes to the environment. Our research focuses on the Effort category of LMA that articulates the dynamic qualities of movement. The historical evolution of Effort and Shape theory, as described by Irmgard Bartenieff, begins with Laban's writings on dance, which are developed through Labanotation, then further expanded through the analysis of Effort elements of factory workers during World War II [1]. The theoretical development continues with the elaboration of Effort and Shape analysis by Laban's students, including Irmgard Bartenieff (ibid). In LMA, Effort is described as: "the dynamic or qualitative aspects of the movement. Dynamics give the feel, texture, tone, or colour of the movement and illuminate the mover's attitude, inner intent, and how they exert and organize their energy. Effort is in constant flux and modulation, with Factors combining together in different combinations of two or three, and shifting in intensity throughout the progression of movement" [42].

Effort encompasses 4 discrete Factors: Weight, Time, Space, and Flow. Each Effort Factor is thought of as a continuum with two opposite ends called elements in which movement can vary and thus reveal different "Effort qualities" [21]. Space is related to the attentional quality to the surrounding environment. One scans the totality of the environment in Indirect Space Effort or investigates a specific focused point in space in Direct Space Effort. Time is related to the quality of acceleration in Quick Time Effort or deceleration in Sustained Time Effort. Weight Effort is related to how one exerts force expressing Strength in Strong Weight or using a delicate intention in Light Weight Effort. Finally, Flow is related to the quality of progression of movement through releasing in Free Flow or controlling the movement in Bound Flow.

In human movement, Effort Factors are normally expressed in combinations and are rarely observed in isolation. Combinations of two Effort Factors are called States and combinations of three Effort Factors are called Drives. In this paper we investigate the modeling of the Basic Effort Actions (BEAs) defined by Laban as a set of eight effortful actions that combine $3 \mathrm{Ef}-$ fort elements from the Weight, Time, and Space Effort Factors (see Table 1) and exclude the Flow Effort Factor. The BEAs definitions were developed by observing the movements of factory workers [21]. Thus, the performance of the BEAs in space can be imagined as the effortful expressive action of pulling factory objects to switch off, turn on, flick over, push, flip, etc. We chose to study these specific BEAs because they are a well-represented sample of actions that cover the range of movement qualities in the Space, Weight and Time Effort continuum, while being precisely articulated and reproducible.

\begin{tabular}{|l|l|l|l|}
\hline Effort Actions & Space & Time & Weight \\
\hline \hline Float & Indirect & Sustained & Light \\
\hline Punch & Direct & Quick & Strong \\
\hline Glide & Direct & Sustained & Light \\
\hline Slash & Indirect & Quick & Strong \\
\hline Dab & Direct & Quick & Light \\
\hline Wring & Indirect & Sustained & Strong \\
\hline Flick & Indirect & Quick & Light \\
\hline Press & Direct & Sustained & Strong \\
\hline
\end{tabular}

Table 1. Overview of the BEAs with the corresponding Effort Factors.

\section{BACKGROUND}

\section{From Movement Expertise to Interaction Design}

Movement-based interaction researchers propose novel approaches in HCI that argue for a aesthetic and embodied appreciation through enriched experiences and attention to the self [39]. We cite among those approaches, move to get moved [19], moving and making strange [27], embodied sketching [30], designing for movement and through movement [44] or somaesthetic design [18]. In these approaches, researchers have emphasized the role of expertise and physical knowledge of the movement in embodied interaction design. Embodied interaction results from the theory of embodiment in which human cognition is a bodily capacity and not just a process of thinking with the brain [20,9]. Over the centuries, dance has built a strong practical and theoretical body of knowledge for performing and crafting movement. This has inspired recent works in HCI to draw upon theories in modern dance [34], and to directly collaborate with experts dancers and choreographers to formalize a movement vocabulary for interaction [11,12]. Other fields, such as Somatics, build acute skills of movement experience and observation. The term somatics refers to body-based practices that use a first-person 
perspective to develop embodied awareness of body sensation and capacity as experienced and regulated from within. For example, Loke and Khut utilize their somatic practice of Feldenkrais methods to design technologies that enable the users to gain awareness of the inner bodily sensation [26]. Recently, researchers explored the benefit of Somaesthetics in designing for the body [18]. This technique involves somatic introspection, meaning "an organized inward-looking inquiry by the individual about his or her bodily perception and its related affective experiences" [41]. Lee et al. used somaesthetics practice to improve the ideation process of interactive product design through a set of movement and design workshops [24].

LMA is a system that focuses on experiencing, observing, and articulating movement patterns. LMA per se is not viewed in the same way as practices that are primarily somatic such as Alexander Technique or Feldenkrais. It has a broader scope because it provides a rigorous use of language to analyze functional and expressive movement based on experiential knowledge. Most interactive systems that incorporate movement qualities rely on LMA's theory of Efforts. Because of the inherent complexity of movement's expressive and qualitative aspects, modeling Effort qualities remains one of the most challenging tasks and an unsolved problem in movement representation and computation.

\section{Computing Movement Qualities}

Some of the earliest work relying on LMA Effort categories in computer animation comes from Norman Badler's research group [4, 46]. For example, Chi et al. developed the EMOTE system that integrates Efforts and Shape categories to animate a 3D character, using mocap data with the aim of producing more expressive and natural simulated movements [7]. Eyesweb platform is another framework using data from video streaming to analyze and classify the expressivity of gestures along Laban Effort Factors [6]. Eyesweb's expressive features approximate Space Factor with the extension and contraction of the limbs and the straightness of movement. Time Effort is considered in terms of duration of the pause and tempo changes. Weight Effort is considered in terms of the amount of tension in movement. Flow Effort is approximated by the shapes of speed and energy curves, the rhythm and the amount of acceleration during motion phases [43].

More recently, Mentis et al. built a system using the Microsoft Kinect for an improvisational dance performance in which audience members' Effort Factors were used to trigger musical events [32]. They collaborated with one CMA to design their Effort model and analyse their Effort observation results. Their Effort features were computed using the position data of the wrist joint given by the Kinect's skeleton. They approximate Strong versus Light Effort by the wrist's position below versus above the hip joint. They approximate Time Effort by a quickly stopped motion versus a continual velocity of the wrist. They approximate Space Effort by detecting a small versus a large deviation of the wrist's trajectory from a straight path. Finally Bound versus Free Flow are approximated by small versus large distance between the wrist joint and the hip joint.

Through capturing one limb with a single accelerometer, Pietrowicz et al. [36] classify Effort Factors and BEAs us- ing Weka machine learning software and simple heuristics to tune parameters of their model. Interestingly, they observed the need to expand their study from a single sensor to multiple sensors for more sophisticated processing of Efforts. Using Pietriwicz et al.'s classifier and additional features of acceleration, trajectory, direction changes and stillness ratio, Maranan et al. developed a prototype based on a single accelerometer's hardware and software called "EffortDetect" to recognize Laban BEAs [29]. Maranan et al.'s EffortDetect does not outperform other Effort recognition systems fed with much richer data. Rather, it assesses the level of BEA recognition that can be accomplished using a single accelerometer.

In these different approaches taken to approximate Effort Factors, we notice a variety of salient features that can be mapped to LMA definitions of Efforts. This variety is due to the inherent limited information available in the commonly used motion capture systems. Nearly all models in the literature focus on capturing one body part and use either data taken from motion capture systems or accelerometers. There is hardly any model combining visual data with bio-signals such as muscle contraction data that could be crucial to model Efforts. We emphasize that our goal is not to criticize the literature. We would like to give credence to the literature attempting to explore movement in technology through the lens of LMA. We acknowledge that the task is challenging because movement is complex and that it is difficult to tease out one aspect of movement alone. Our literature review positions our own computational approach in a broader design perspective that argues for a multimodal acquisition and capture of the whole body. Moreover, we take a participatory method integrating CMAs expertise and we propose a quantitative evaluation of our Effort recognition system.

\section{Observing Movement Qualities}

LMA's inter-rater reliability among expert observers has been assessed in early works of psychologist Martha Davis [8]. Stevens et al also showed a significant inter-rater reliability of the Effort Factors on observation of curated video specimen of solo Marimbists' bodily expression [5]. These studies have shown that LMA can be considered as a reliable expert observational model of movement qualities. On movement qualities observation, Mentis el al's paper showed that movement qualities can be seen differently using the LMA lens depending on experience and background of a viewer [32]. Moreover, the way in which one calls on experiences and embodied enaction to construct their observational skills affect their capacity to accurately see and define Efforts. They point out to one of the main principles in LMA observation, that is the need to embody a movement quality before seeing it. Their study does not argue that LMA is subjective but rather that its reliability depends on the training in movement observation and the knowledge of LMA. Thus, movement observation is challenging and requires substantial training to build embodied skills in addition to understanding the LMA language.

Similarily, Fdili Alaoui et al found that embodiying movement is necessary in order to observe it, in both interaction design and LMA observation practice. They observed 12 CMAs in their movement observation practice [13]. They showed that 
CMAs use their own bodily sensations in the analysis of another's movement through kinesthetic empathy. They also showed that CMAs encourage group observation because it allows them to open to others' perspectives and to extend their observational skills. In group, CMAs seek for consensus in order to insure the reliability of their observation. Fdili Alaoui et al also interviewed HCI researchers on how they observe movement in their design process [14]. They articulated three techniques for performing movement observation inspired by somatics and movement studies: attunement, attention, and kinesthetic empathy. These techniques emphasize the role of embodying movement before seeing it, and the role of movement literacy in movement observation in HCI. We built upon theses findings to run an interview that insures ecological conditions for observing Efforts and to design a model informed by such a reliable observation. Thus, we contribute to designing movement-based interactions by articulating Efforts and making precise choices of sensors and computational modeling of movement qualities.

\section{MODELLING EFFORTS FROM EXPERTS' OBSERVATION}

In order to integrate LMA expertise into the computational model of Efforts, we interviewed two CMAs from the Laban Institute of Movement Studies. Our research goal was to elicit "how" they observe Efforts and articulate the visual and kinesthetic cues used in their observational process. We selected a structured interview format, in which the discussion was shaped by "mining" the experts' comments in order to allow new ideas to emerge through our exchanges. This process drove us to the selection of an appropriate set of multimodal sensors and the design of high-level movement features that correlate with the Effort Factors of Weight, Time and Space that compose the BEAs.

\section{Protocol and Apparatus \\ Participants}

We interviewed two senior CMAs. They each bring over 20 years of LMA expertise, experience and collaboration in movement observation and analysis. They train LMA students and direct the LMA training programs all over the world. They have collaborated with each other in the most impactful observational projects involving for example making movement portraits of politicians. Even though the interview was performed with only 2 CMAs, they are world-renowned experts in LMA and their observation of movement qualities is consistent. It represents a reliable ground and corresponds to that used around the world by the community of CMAs. Our interview was an ecological study of how these CMAs perform movement observation together using video. Their participation in the interview together is faithful to their group observation practice as shown by Fdili Alaoui et al to allow for achieving consensus [13].

\section{Experimental procedure}

During the interview, we displayed a video of a phrase with the 8 BEAs recorded during a Motion Capture session with a dancer who has undertaken an LMA certification program. The video was validated as accurate by another CMA. Neither the dancer nor the CMA who performed and validated the sequences participated to our interview. We chose to display one video of one expert mover rather than random videos because we targeted all the Efforts actions in LMA. Moreover, from the LMA perspective, the BEAs, when performed by a CMA and subsequently validated as accurate by another CMA, can then be unambiguously recognized with no significant variations across any other number of CMAs. This is also corroborated by previous studies cited in our related works [5].

During the interview, the 2 senior CMAs talked out loud while watching the video, describing "what" they observed and "how" they observed it. They also reported on the visual and kinesthetic cues that they were applying to their Efforts observation. As shown in [13], CMAs sought for consensus throughout their discussion. For that purpose, the 2 CMAs negotiated with each other. This implies compromising to another's opinion if her rationale was convincing or attempting to convince the other by finding arguments to support the observation. We videotaped and audio recorded the 2-hour interview.

\section{Data analysis}

We analyzed the recorded data from the interview based on a qualitative coding approach that aims to create knowledge both from the viewer and the viewed. This allowed us to build an interpretative understanding of the participants' meanings in order to elicit relationships among emerging concepts. We followed guidelines inspired by the process described in [45] and coded the audio and video recordings at three different levels (open-coding, axial-coding and selective-coding) which enables a story or narrative to emerge from the research concepts.

\section{Findings \\ How LMA experts make decisions}

Movement is complex by nature. According to CMAs: "There is almost in all cases a multitude of things happening. And it is difficult to isolate one aspect alone. This is why the confusions occur" [citation from transcription]. CMAs simultaneously used multiple cues to make a decision about the most significant component of LMA observed. Because of the complexity of the decision-making process, the most significant components observed usually emerged from a discussion and an agreement across the CMAs. In most cases, the agreement among CMAs was achieved, indicating the reliability of the observational cues and suggesting their replicability in a computational model.

\section{Multimodal observation}

CMAs argued for the combination of multiple movement sensing modalities through the collection of positional, dynamic and physiological measures. The CMAs considered that in order to translate their kinesthetic and visual observation cues faithfully into higher-level features correlated to the LMA Effort concepts, the first step is to investigate multiple modalities of sensing motion. Thus, our selection of sensors is as diverse as the visual and kinesthetic cues used by expert CMAs to observe Effort patterns.

\section{How LMA Experts Observe Weight Effort}

Video seem to diminish the CMAs ability to perceive Weight Effort. CMA A considers that "Weight is the most elusive thing to capture in video". In order to make a decision about 
the mover's intention towards Weight from video streams, the CMAs reported that they empathetically interpolate Weight Effort from bodily organization and sense of forcefulness, as well as the use of the floor, or the use of breath support. From this statement we inquired that sensors such as Electromyograms (EMGs) giving a sustained contraction versus release of the muscle could be mapped to the variation of force used in Strong versus Light Weight Effort. The CMAs agreed that they correlate with the Weight Effort. However, CMAs acknowledged the limitation of the use of muscle contraction for Weight Effort Factor is that "on a physiological level there is a connection to the muscular system for both increasing and decreasing pressure (Weight Effort) and for controlling or releasing (Flow Effort). The mover can also experience Flow through muscular control and release of the upper arms, and experience Weight through the muscular response towards gravity". To overcome this limitation, we opted for EMGs placed on the mover's two forearms to characterize Weight Effort. We used the Infusion Systems BioFlex V1.3 dry electrode, along with a platform based on a central wireless module sending data over the "Zigbee" protocol to the host computer. We extracted the envelopes of the EMG data obtained by Bayesian filters [38] that distinguish the contraction from the release of the muscle tension and thus the Strong from Light Weight Effort.

\section{How LMA Experts Observe Time Effort}

CMAs observe Time Effort through the patterns of change in movement acceleration and deceleration. CMAs legitimated features of Time Effort computing the variations in acceleration and deceleration of the limbs motion. Therefore, we propose to use the norm of jerk of the motion to distinguish Quick Effort from Sustained Effort. This metric is computed as the magnitude of the derivative of the acceleration value. We used a wireless 6-DOF inertial measurement unit called Modular Musical Objects (MOs) [37] placed on the mover's right wrist. The $\mathrm{MO}$ is small module that contains $6 \mathrm{DOF}$ inertial sensors (3D accelerometer and 3D gyroscope) and relies on a platform based on a central wireless module. The wireless protocol is based on the IEEE 802.15.4, generally called "Zigbee".

\section{How LMA Experts Observe Space Effort}

CMAs consider that video does not carry enough cues to contribute to the observation of the mover's attention to Space. They reported that it is even more limited in terms of accessing the mover's intent toward Indirect Space Effort. Video does not allow viewer to observe the mover's exploration of the whole environment through his whole body, including the consciousness of the space behind her. When observing Indirect Space Effort from Video footage, CMAs reported that "the CMAs are picking up kinesthetically something about the movers accessing a sensory gathering that their entire body sees with the back surface of their body. This is very limited in looking at a video" [citation from transcription]. In addition to these kinesthetic cues, the mover's gaze (not necessarily visible on video) has been reported by the CMAs as being a good indicator of the mover's intention toward Space Effort. For example, CMAs reported that observing a mover closing his eyes indicated that he is not in Space Effort.
To computationally characterize Indirect versus Direct Space Effort, CMAs suggested considering Laban Shape qualities of Spreading and Enclosing and their respective affinities. Indirect Effort correlates with the Spreading quality that requires an extension and opening of the body and thus an increase in the distance between the elbows and the chest. Direct Effort correlates with the Enclosing quality that requires a contraction and thus a decrease in bodily opening and the distance between the elbows and the chest. We compute a feature of extension and contraction as an average of the variations of the distances between the chest, and the elbows. Such feature is also used by Volpe in the Eyesweb platform [43]. We compute these rates using positional data of the chest and elbows from 3D mocap. We used the marker-based 3D mocap studio at Emily Carr University of Art and Design in Vancouver, a system composed of 20 Vicon T160 cameras and 20 Vicon MX40 cameras with a sampling rate of $120 \mathrm{fps}$. We used a configuration of 57 markers for the 3D Vicon mocap, set on the body of the movers by fixing 5 markers on each foot and ankle, 4 on each leg, 2 on each shoulder, 4 on each arm, 4 on each hand, 6 on the hips, 4 on the spine, 4 on the chest, and 5 on the head.

\section{EVALUATING MOVEMENT FEATURES}

We integrate the results of our interview to compute a set of features for each type of data and each Effort Factor:

Positional data: Space Feature variation of the distance between the right elbow and the chest obtained from the 3D Motion Capture.

Dynamic data: Time Feature norm of the jerk extracted from the accelerometer placed on the right wrist.

Physiological data: Weight Feature estimation of the muscle activation from the EMGs using a non-linear Bayesian filter.

In this section, we will evaluate our features by examining their profiles on a set of movement data. We also evaluate our features' performance for a task of real-time Effort recognition using a machine learning algorithm. We compare the features' recognition rates for the BEAs and for each Effort Factor. The ground truth for the recognition algorithm is the experts' annotation of the movement data.

\section{Data Collection}

The results of the interview support our choice of multimodal sensors, their number and their disposition on the body. The multimodal measures bring together different types of data: positional data is acquired though mocap, dynamic data is obtained with inertial sensors, and physiological data is recorded with EMGs. We used a sophisticated 3D Vicon Motion Capture system (Mocap), 2 EMGs and 1 accelerometer for data acquisition. We ran a multimodal data acquisition session with 2 expert CMAs that were not part of the interview: one male and one female aged between 40 and 55 years old that both have over 15 years of experience in LMA. We ask them to perform and vocalize the BEAs in the following sequences:

- 12 trials of a movement phrase composed of 8 segments, one for each BEA in the order defined by Table 1. 
- For each BEA, we recorded 3 trials of a movement phrases composed of 8 segments of the Action performed repeatedly in different spatial configurations. We recorded these sequences to be able to evaluate our model on the BEAs in various orders and spatial configurations.

We collected in total 12 sequences of 8 spatially ordered BEAs and $3 \times 8$ sequences of 8 non-ordered BEAs for each performer.

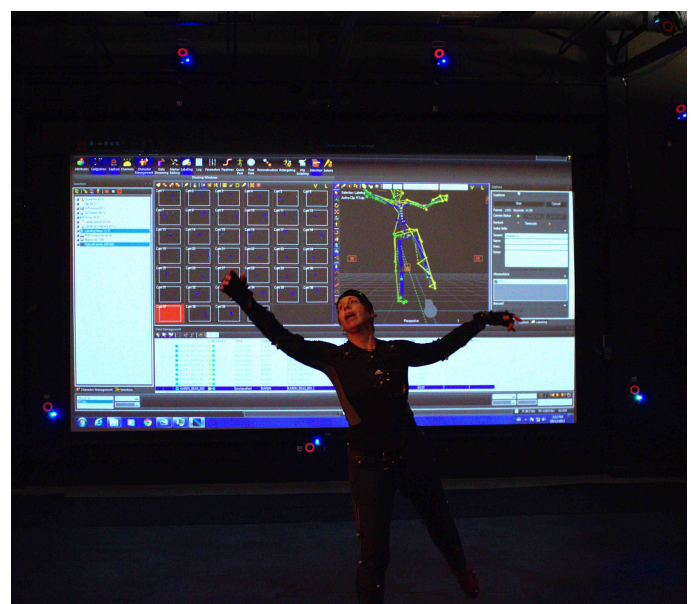

Figure 1. The data collection using EMGs, an accelerometer and a Vicon Motion Capture

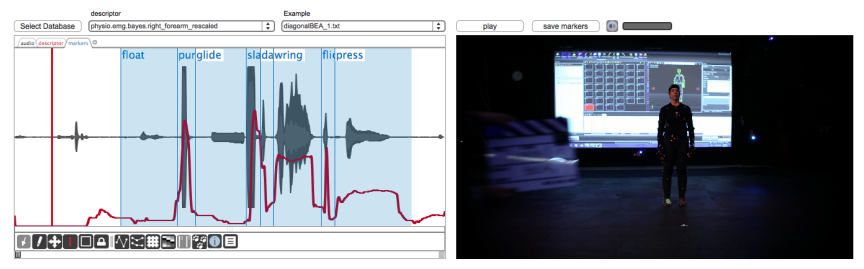

Figure 2. The data annotation interface using MuBu.

\section{Data Annotation}

We built the annotation interface (showed in Figure 2) in Cycling'74 Max6 using the MuBu library ${ }^{1}$ [40] in order to be able to browse the recorded collection of movement data and to enable synchronized playback of data streams from different multimodal sensors or high-level features with the audiovisual streams. The interface allows for manual data annotation through the definition of labeled segments. We used this interface collaboratively in order to observe the videos and the sensors' data of the collected movement sequences. The first author of the paper who is also a CMA, manually segmented each recorded movement sequence into unitary BEAs and annotated these segments by uploading a label into the data using our customized interface. These annotations are the ground truth to which our automatic Effort recognition outputs is compared.

\section{Examining Features' Profiles Weight Effort Feature}

Figure 3 shows that the muscle contractions feature exhibit distinct profiles with higher envelope in the BEAs with Strong
Weight than in the BEAs with Light Weight. Moreover, these profiles are shown to be consistent across trials in Figure 4. This suggests that the envelope of the EMGs consistently correlates the contraction versus release of the muscles with the Strong versus Light Weight Effort.

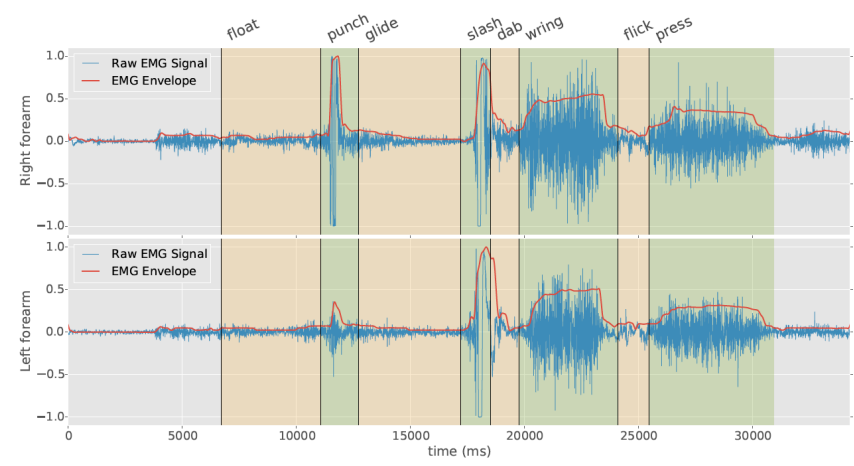

Figure 3. Normalized envelope of the EMG of the right and left forearms, on a sequence of BEAs. The movement phrase is segmented and annotated manually by the CMA into the 8 BEAs, and segments are highlighted according to the Weight Effort Factor (Yellow: Light, Green: Strong)

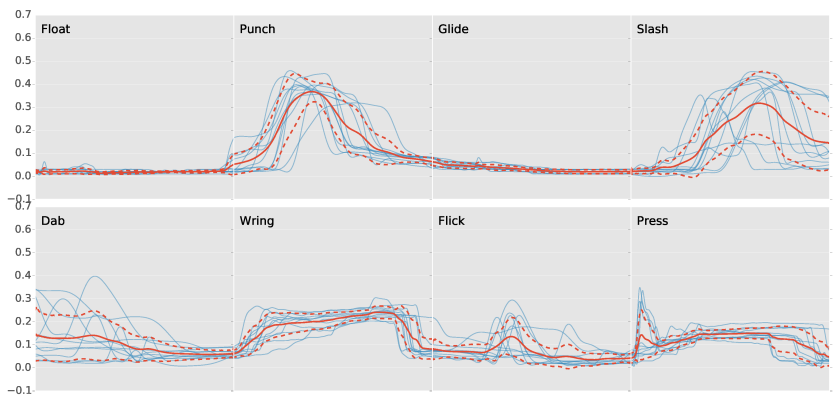

Figure 4. Superposition of the EMGs envelopes, for 12 trials of BEAs of one performer. Examples are realigned and time is normalized for each BEA.

\section{Time Effort Feature}

Figure 5 illustrates the distinguishable profiles of the norm of the jerk with a peak for Quick Time versus a flat for Sustained Time, on one example of the BEAs phrase. These profiles are shown by Figure 6 to be consistent across trials which suggest that the jerk consistently correlates with the Time Effort elements of Quick and Sustained.

\section{Space Effort Feature}

Figure 7 illustrates the profiles of the Space Effort feature for one phrase with eight BEAs. Although Figure 8 shows consistency across trials, Figure 7 shows that the profiles of the resulting Space feature do not demonstrate clear patterns distinguishing Direct from Indirect Space Effort. This is related to the limitations in approximating Space Effort with Shape qualities. Space Effort as defined in LMA is in essence challenging to measure computationally because it is about the mover's attention to space, rather than his actual movement configuration in space or bodily organization. 


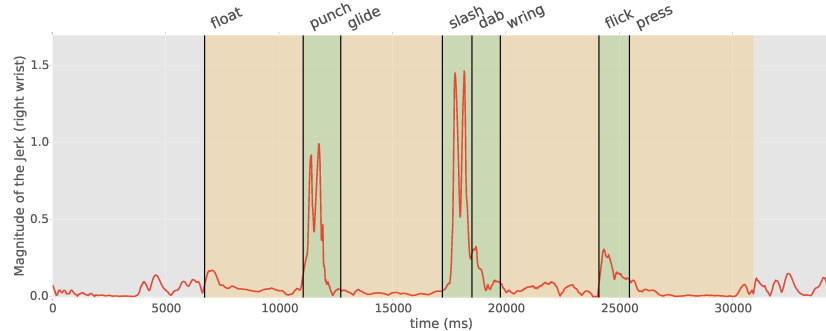

Figure 5. Norm of the jerk computed from data from the accelerometer placed on the right wrist, on a sequence of a BEAs. Segments are highlighted according to the Time Effort Factor (Yellow: Sustained, Green: Quick)

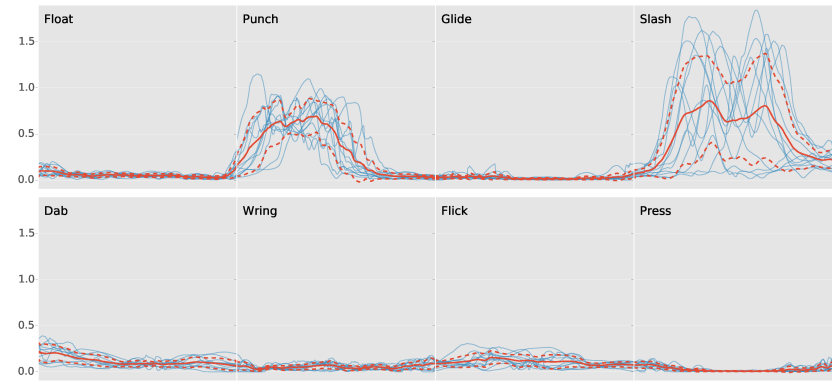

Figure 6. Superposition of the Jerk magnitude from the accelerometer placed on the right wrist, for 12 trials of BEAs of one performer.

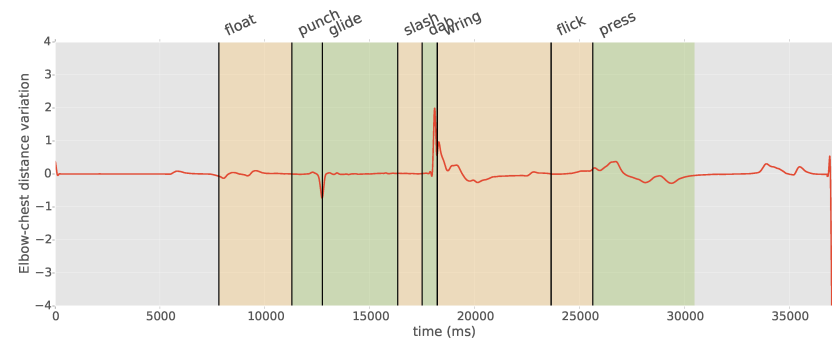

Figure 7. Variation of the distance between the chest and right elbow markers tracked by mocap, on a sequence of BEAs. Segments are highlighted according to the Space Effort Factor (Yellow: Indirect, Green: Direct)

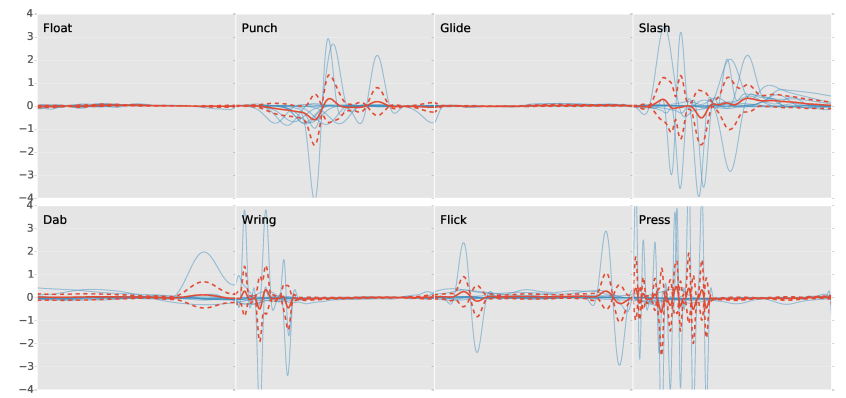

Figure 8. Superposition of distance between mocap markers of chest and right elbow, for 12 trials of BEAs of one performer.

\section{Evaluating Features for Effort Recognition}

We evaluate our features's performance on a task of Effort recognition. Our focus on Effort recognition concerns appli- cations with expressive movement-based interactions. Hence, we stress the importance of moving from the simple recognition of discrete movement patterns to continuous recognition methods that quantify movement qualities in real-time.

\section{Continuous Effort Recognition using HMMs}

Our recognition method is dedicated to continuous interaction, and focuses on the real-time recognition of Effort qualities. Instead of performing offline classification on a set of segmented gestures, our machine learning model continuously estimates the likeliest Effort, at each time step and causally - i.e. using the partial observation sequence up to the current frame.

We used a recognition algorithm based on Hierarchical Hidden Markov Models (HHMMs) [15], in which each Effort element is modeled by a 5-state HMM with a left-right topology encoding the temporal dynamics of the movement features. The Effort models are linked by a higher-level ergodic transition structure allowing equiprobable transitions between models. As in the case of Gesture Follower [2], the HHMMs can train each model from a single example. The system uses an Expectation-Maximization algorithm adapted to converge quickly with few training examples, as required by real-time applications in the context of the interactive arts. The testing phase consists in evaluating the likeliest Effort for each frame of the test sequence. This is performed in real-time using a forward algorithm that continuously estimates the likelihood of each Effort model. The HHMM library ${ }^{2}$ is available online and comes with Python bindings, and is also implemented as a set of externals for Cycling'74 Max6 using the MuBu library.

\section{Feature Evaluation by Cross-Validation}

Two aspects of the recognition are evaluated: the recognition of the BEAs, and the recognition of the isolated Effort Factors of Time, Weight, and Space. Our evaluation metric of the movement features is based on a comparison of the recognition scores obtained by cross-validation, according to the following protocol:

1. The performances are manually annotated and segmented by a CMA among the authors from the video recordings.

2. Iteratively, for each single performance, we use all of its segments to train the Effort models.

- When the task is to recognize the 8 BEAs, we use the 8 segments of a single performance to train the BEAs' models.

- When the task is to recognize the 2 elements of a single isolated Effort Factor - e.g. for Time Effort Factor, Quick versus Sustained, - we use the 8 segments of a single performance to train the corresponding Effort elements' models.

3. The recognition is performed over all the other performances that are not used for the training phase.

For each test sequence, the model outputs the corresponding sequence of recognized Efforts for each frame. Considering our focus on continuous recognition, we evaluate the quality of the recognition as the percentage of frames accurately

\footnotetext{
$2_{\text {https: //github.com/Ircam-RnD/xmm }}$
} 
recognized rather than the number of accurately classified presegmented gestures in a test example. The ground truth here is the expert's segmentation and annotation of the movement data. Our frame-based recognition rates are more relevant to our model that output the recognized Efforts frame by frame. They can be understood as the total amount of time during which the algorithm is effectively recognizing the Efforts in the sequence rather than a discrete number of correctly identified segments. It reveals the quality of the recognition as a continuous process. In the following sections, the result scores represent the average of the percentage of correctly labeled frames over all the training and test examples according to experts annotations. We compare 5 combinations of features: (A) elbow-chest distance variation; (B) norm of the jerk (right wrist); (C) envelope of the EMG (right forearm); (D) combination of the three features; (E) combination of the three features + speed \& acceleration.

\section{Results: Comparing Movement Features}

In this section we compare the results of the recognition for all the high-level features elaborated from the participatory design process.

\section{Recognition of ordered BEAs}

We report on the evaluation of the recognition of the BEAs ordered in the order defined by Table 1, from the 12 trials of a movement phrase with all 8 BEAs of one of the performers. Figure 9 reports the results of the recognition of the BEAs and each of the Weight, Time, and Space Factors using the highlevel features considered independently or combined. Note that the chance level for BEA recognition is $12.5 \%$ and that the chance level for isolated Efforts recognition is $50 \%$.

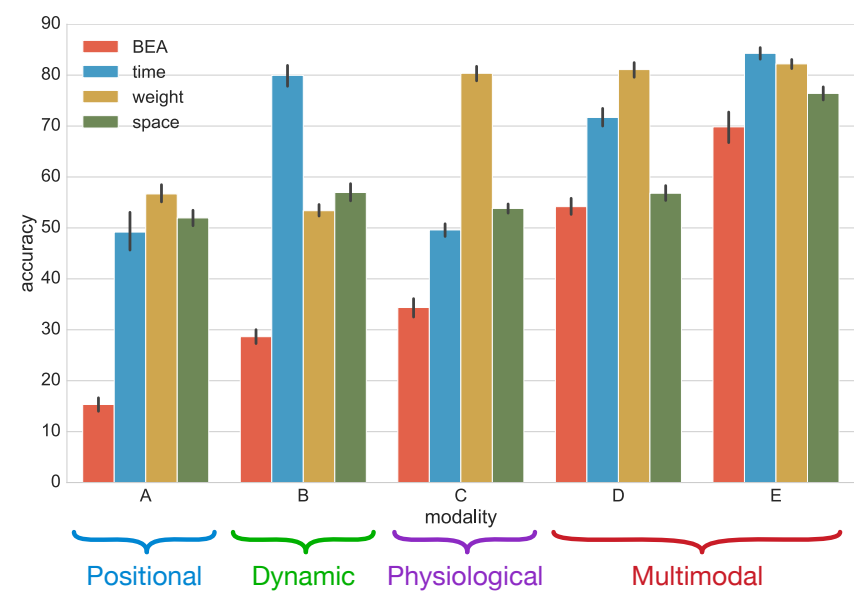

Figure 9. The overall results of the recognition of the BEAs and their three Effort Factors, depending on the various combinations of highlevel features. $A$ : elbow-chest distance variation, $B$ : norm of the jerk (right wrist), $C$ : envelope of the EMG (right forearm), $D$ : combination of the three features, $E$ : combination of the three features + speed \& acceleration

The results confirm the relevance of the features designed for the Time and Weight Effort Factors. Indeed, the best result for the recognition of the Time Effort is obtained with the dynamic feature of the norm of the jerk (B) with $80 \%$ accuracy. With one-way ANOVA, we found a significant effect of the features on the recognition accuracy $(F(4,655)=221$, $p<0.001$, partial=0.57). A Tukey's pairwise comparison revealed the significant differences between $B$ and $A, C, D$ $(p<0.01)$, but no significant difference was found between $\mathrm{B}$ and $\mathrm{E}$.

The recognition rate of the Weight Effort is significantly higher for the physiological feature of the EMGs envelope (C) with $80 \%$ accuracy. With one-way ANOVA, we found a significant effect of the features on the recognition accuracy $(F(4,655)=$ 437, $p<0.001$, partial=0.73). A Tukey's pairwise comparison revealed the significant differences between $\mathrm{C}$ and A, B $(p<$ 0.01 ), but no significant difference was found between $C, D$ and $\mathrm{E}$.

However, the feature designed for the Space Effort Factor did not perform significantly better than other features. With one-way ANOVA, we found a significant effect of the features on the recognition accuracy $(F(4,655)=198, p<0.001$, partial $=0.55)$. A Tukey's pairwise comparison revealed the significant differences between the multimodal features $E$ and A, B, C, D $(p<0.01)$. This result reveals that the addition of speed and acceleration information helped recognize the spatial directionality rather the mover's attention towards the environment in Space Effort. This result correlates with the interview with the CMAs that revealed the difficulty to characterise Space Effort from positional data and the limitation reported in the Eyesweb system [43].

Finally, when comparing the recognition rates of each of the high-level features, alone, they do not seem to distinguish the 8 BEAs, with a maximum of $34 \%$ accuracy achieved with the physiological measure. However, the recognition rate of the BEAs is significantly increased when combining all of these high-level features into a multimodal measure with $54 \%$ accuracy. Moreover, these results improve consistently when combining the high-level features with the dynamic measurements of speed and acceleration of the right wrist with $69 \%$ accuracy. With one-way ANOVA, we found a significant effect of the features on the recognition accuracy $(F(4,655)=$ $455, p<0.001$, partial=0.73). A Tukey's pairwise comparison revealed the significant differences between all conditions under $p<0.01$.

Note that the speed and acceleration measurements are sensitive to the movement orientation in space. Therefore, their optimal recognition rates of the BEAs might be highly correlated with the recognition of the movement's spatial configuration in the order in which the Actions were recorded.

\section{Recognition of non-ordered BEAs}

In this section, we report on the evaluation of the recognition of the BEAs not affined in space. Again, we train the Effort models with one sequence of the BEA affined in space. We then evaluate the recognition of Effort on the 3 trials of each of the 8 BEA performed by one of the performers repeatedly in various spatial configurations.

Figure 10 reports the results of the recognition based on the high-level features elaborated from the participatory design process. The recognition scores support the relevance of the features designed for the Time and Weight Effort, even when 


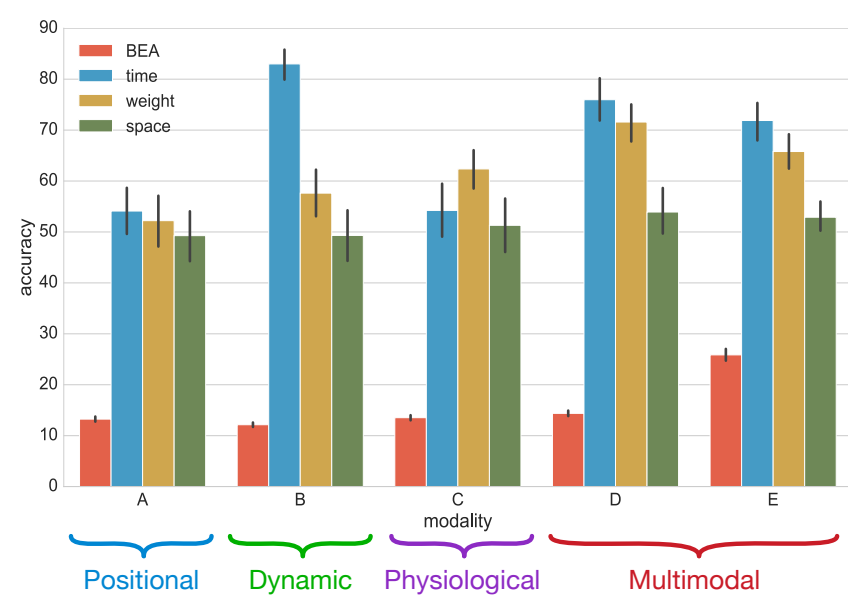

Figure 10. The overall results of the recognition the BEAs, not ordered and their three Effort Factors, depending on the various combinations of measures and High-Level Features. A: elbow-chest distance variation, $B$ : magnitude of the jerk (right wrist), $C$ : envelope of the EMG (right forearm), $D$ : Combination of the three features, $E$ : Combination of the three features + speed $\&$ acceleration

these Efforts are performed not in order and in different spatial configurations. Indeed, the dynamic feature of the magnitude of the jerk provides significantly better recognition rate of the Time Effort with $84 \%$ accuracy. This effect was found significant with one-way ANOVA $(F(4,1435)=36.8, p<0.001$, partial $=0.09)$, and Tukey's pairwise comparison revealed the significant differences between B and A, C, E $(p<0.01)$. Interestingly, combining all the features leads to a decrease of the recognition of the Time Effort, although the effect is not significant. This highlights interactions of the additional modalities with the dynamics feature, and therefore supports the relevance of the magnitude of the jerk as a descriptor of the Time Effort. At the contrary, the combination of high-level features outperforms the proposed high-level feature of Weight with $73 \%$ accuracy, for only $62 \%$ with the physiological feature only. This effect was found significant with one-way ANOVA $(F(4,1435)=11.8, p<0.001$, partial $=0.03)$, and Tukey's pairwise comparison showed that combined features $\mathrm{D}$ and $\mathrm{E}$ performed significantly better than A, B, C $(p<0.05)$. However, the feature designed for the Space Effort Factor does not increase the Space Effort recognition in comparison with other features or measurements.

Finally, when evaluating the recognition of the BEAs performed without the spatial configuration nor order, all the measurements and the features seem to poorly recognize them. This confirms that the previous rates of recognition of the BEAs achieved by dynamic and positional measurements seem to be highly related to the recognition of the movement spatial direction rather than the Effort intent.

\section{IMPLICATIONS FOR DESIGN}

Our contribution builds upon previous works in designing for movement qualities as interaction modality [12]. We make available a computational system for real-time recognition of LMA Efforts that can be directly applied to HCI applications exploiting accelerometer, EMGs and motion capture system.
Our platform can adapt to consumer devices such as Myos, Kinect infra-red cameras or ubiquitous accelerometers in handheld mobile phone as well as wearable on-body devices. Our system enables these applications to integrate users' movement qualities and thus expand the affordances of their movement modality beyond the solely functional ones. While our system utilizes all three sensors of accelerometers, EMGs and motion capture, it can also be trained with fewer sensors. Depending on the configuration, Time, Weight or Space Efforts can be selectively characterized. For example, it has the potential to be ported to existing mobile devices by transmitting its accelerometer data into our recognition engine and mapping Time Effort recognition to higher-level outputs used by the application.

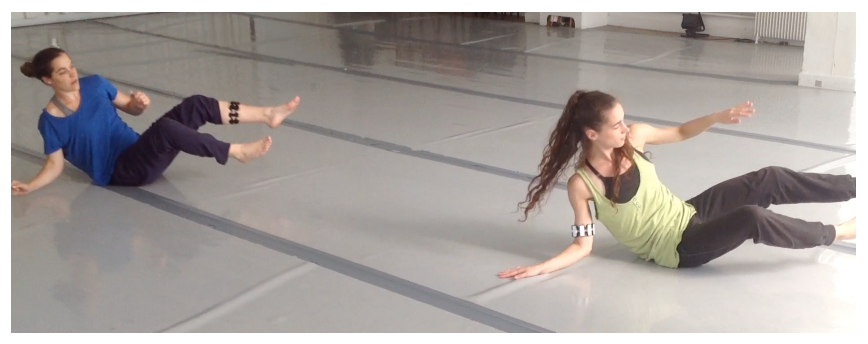

Figure 11. Rehearsals with the two dancers of the performance equipped with the Myos training the Effort recognition system

We illustrate how we integrated our approach to design expressive movement-based interactive systems for dance. In particular, we shortly report on three applications using the Effort recognition. We describe 1) a dance performance, 2) a participatory design workshop with dancers and choreographers and 3) a pedagogical workshop for dancers. All three interactive applications are based on our model of Time and Weight Efforts, because they are the most accurately characterized using the proposed sensors and features.

Our first application is a dance piece, where two dancers are equipped with $\mathrm{Myos}^{3}$ that provide both accelerometers and EMG data. Dancers perform 8 different dance sequences of 10 seconds combining Light versus Strong Weight Effort and Quick versus Sustained Time Effort. We trained our system offline with the 8 dance sequences. During the performance on stage, the system recognizes the dancers' Effort qualities and continuously estimates in real-time the associated dance sequence that is most likely to be performed. We then created a simple mapping where the system's decision triggers videos that have been pre-recorded with the two dancers performing the exact dance sequences in another context: a countryside home with a natural environment. This interaction was built according to the artistic intention of the piece that aimed at creating a shift in the time and space of the performance allowing to disrupt the viewers attention to the stage.

We applied the same approach to a participatory design workshop with 4 dancers and a choreographer in preparation of a future collaboration together. Dancers, equipped with Myos, trained the system on dance sequences of their choice combining Time and Weight Efforts. During the performance,

\footnotetext{
${ }^{3}$ https://www.myo.com/
} 


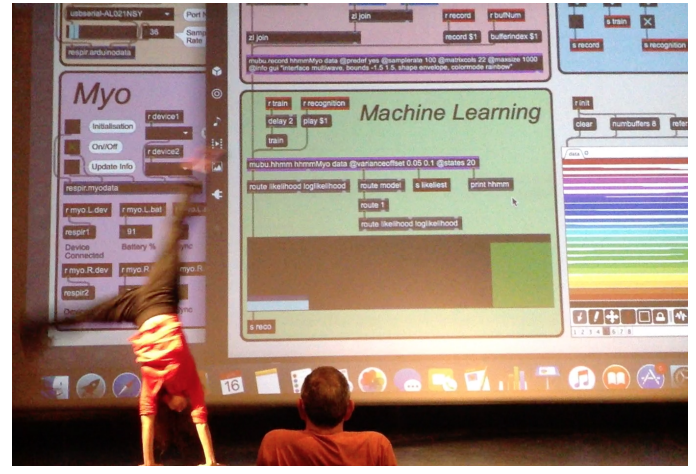

Figure 12. Participatory design session with dancers equipped with the Myos improvising with the Effort recognition.

the system continuously estimates in real-time the movement quality and displays the recognition rate of the dance sequence. As shown in figure 12, dancers improvised and integrated the dance sequences while exploring the interaction with the system and observing the system's response in terms of recognition rates. We then run an informal brainstorming session in order to explore possible interaction scenarios using the Effort recognition engine in our future piece.

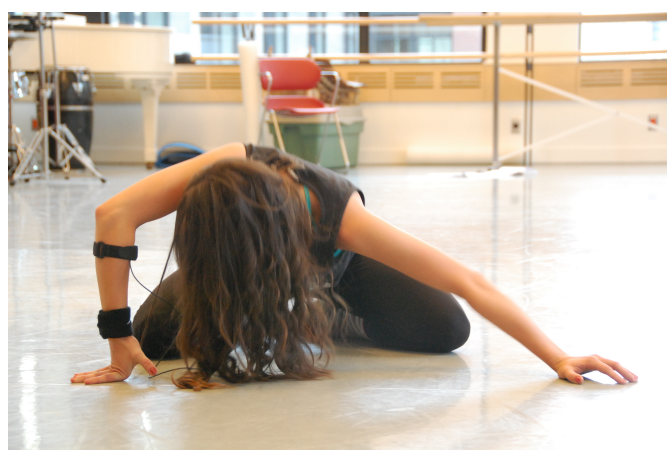

Figure 13. Pedagogical workshop with dancers equipped with an EMG and an accelerometer improvising with the Effort sonification.

Finally, we applied our system to provide sound feedback that guides the performance of Effort qualities in a pedagogical context [16]. As shown in figure 13, dancer were equipped with accelerometers and EMGs. They were guided by a CMA in experiencing Time and Weight Efforts through improvising with a sonic feedback. We synchronously pre-recorded CMAs' vocalizations and performance of Efforts to form a set of multimodal sequences. During the Training phrase, the system learns the relationship between the Effort features and the voice features. The system is then used for interactive sonification: During the Performance the dancers' live Effort qualities are recognized by the system that continuously synthesizes the associated pre-recorded vocalizations.

From all our applications, we were able to test our Effort recognition system and observe dancers' response in different context. Our observations allow us to assess the potential of our system for supporting a rich interaction with video, sound or any other medium, that integrates a more expressive modality as well as a better articulation of Efforts. Our work with professional dancers have showed how our technology allows to experience Efforts through providing a higher-level movement features and richer computational models while adapting to a variety of artistic and expressive movement practices and contexts. Our system was successfully used in applications in dance but shows also future potential to be expanded into the greater HCI applications for health and well-being, gaming, the quantitative self and entertainment.

\section{CONCLUSION}

We contribute to articulate the value of expert movement knowledge for embodied interaction design. We undertake a participatory design perspective with Certified Laban Movement Analysts to unpack their Effort observation process alongside human-centred machine learning strategies [17]. Informed by the multi-sensory process of Effort observation, we selected a set of multimodal sensors, providing positional (motion capture), dynamic (Inertial sensors) and physiological information (electromyography) and designed computational features of Efforts. The evaluation of each feature on a task of real-time Effort recognition emphasizes that Time Effort is better represented with the dynamic feature of the jerk norm from inertial data, and that Weight Effort is better conveyed through physiological measures such as muscle activation. These results confirm the value of grounding computational system design in expert knowledge. Nonetheless, the feature designed for the Space Effort, measuring the body's quality of Spreading/Enclosing, did not prove as efficient to characterize fully Space Effort. This results underlines the inherent difficulty to characterize LMA concept of Space Effort. The current computational approaches to Space Effort need further delineation in order to truly characterize users' expression through Spatial attention.

Our study shows that in order to characterize Laban Efforts, one needs rich multimodal data, combining positional, dynamic and physiological information. Limiting the sensing modalities means limiting the number of movement qualities to be integrated in the interaction. Thus, such considerations should be taken into account in designing the expressive scope of a movement quality based interactive system.

Finally, we provide a reflection on how expert-informed movement features can contribute to interaction design through the notion of movement quality [12]. Going beyond the purely functional aspect of movement to describing "how" the movement is performed. This is momentous in enriching the aesthetic experience of users [35]. By expanding movement recognition to include the nuanced language of movement qualities, our approach has a strong potential for movementbased interactions with applications in the arts, digital media, entertainment, education, or rehabilitation. Because we can leverage on human's most valuable richness that is their movement expression. Because "which is connected with the mastery of movement, remains fundamental as long as the human race exists." Rudolf Laban.

\section{ACKNOWLEDGMENTS}

This research is part of the movingstories project, financially supported by the Social Sciences and Humanities Research Council of Canada (SSHRC partnership grant GT 15152). 


\section{REFERENCES}

1. Irmgard Bartenieff. 1970. Four Adaptations of Effort Theory in Research and Teaching. Gordon and Breach Science, New York, USA.

2. Frédéric Bevilacqua, Bruno Zamborlin, Antony Sypniewski, Norbert Schnell, Fabrice Guédy, and Nicolas Rasamimanana. 2010. Continuous realtime gesture following and recognition. In Embodied Communication and Human-Computer Interaction, volume 5934 of Lecture Notes in Computer Science. Springer, 73-84.

3. andreas Scharader Borge Kordts, Bashar Altakrouri. 2015. Capturing and Analysing Movement Using Depth Sensors and Labanotation. In ACM SIGGRAPH Symposium on Engineering Interactive Computing Systems.

4. Durell Bouchard and Norman Badler. 2007. Semantic segmentation of motion capture using laban movement analysis. In Intelligent Virtual Agents. Springer, 37-44.

5. Mary C. Broughton and Catherine J. Stevens. 2012. Analyzing Expressive Qualities in Movement and Stillness: Effort-Shape Analyses of Solo Marimbists' Bodily Expression. (2012).

6. Antonnio Camurri, Barbara Mazzarino, Matteo Ricchetti, Renee Timmers, and Gualtierro Volpe. 2004. Multimodal analysis of expressive gesture in music and dance performances. In Gesture-based communication in human-computer interaction volume 2915 of Lecture Notes in Artificial Intelligence. Springer, 357-358.

7. Diane Chi, Monica Costa, Liwei Zhao, and Norman Badler. 2000. The EMOTE model for effort and shape. In In Proceedings of ACM SIGGRAPH. ACM, 173-182.

8. Martha Davis. 1987. Steps to achieving observer agreement: the LIMS reliability project. Movement Studies (New York: Laban / Bartenieff Institute) 2 (1987), 7-19.

9. Paul Dourish. 2004. Where the action is: the foundations of embodied interaction. The MIT Press.

10. Daniel Fallman. 2011. The new good: exploring the potential of philosophy of technology to contribute to human-computer interaction. In Proceedings of the 2011 CHI Conference on Human Factors in Computing Systems (CHI'11). ACM, 1051-1060.

11. Sarah Fdili Alaoui, Frederic Bevilacqua, Bertha Bermudez, and Christian Jacquemin. 2013. Dance Interaction with physical model visualization based on movement qualities. International Journal of Arts and Technology, IJART (2013), 0-12.

12. Sarah Fdili Alaoui, Baptiste Caramiaux, Marcos Serrano, and Frédéric Bevilacqua. 2012. Movement qualities as interaction modality. In Proceedings of the ACM SIGCHI Conference on Designing Interactive Systems (DIS'12). ACM Press, Newcastle, UK, 761-769.
13. Sarah Fdili Alaoui, Kristin Carlson, Shannon Cuykendall, , Karen Studd, Karen Bradley, and Thecla Schiphorst. 2015a. How Do Experts Observe Movement. In Proceedings of the International Workshop on Movement and Computing. Vancouver, BC, Canada.

14. Sarah Fdili Alaoui, Thecla Schiphorst, Shannon Cuykendall, Kristin Carlson, Karen Studd, and Karen Bradley. 2015b. Strategies for Embodied Design: The Value and Challenges of Observing Movement. In Proceedings of ACM Creativity and Cognition (CC '15). ACM, Glasgow, Scotland, UK, 121-130.

15. Jules Françoise, Baptiste Caramiaux, and Frédéric Bevilacqua. 2012. A Hierarchical Approach for the Design of Gesture-to-Sound Mappings. In Proceedings of SMC conference. 233-240.

16. Jules Françoise, Sarah Fdili Alaoui, Thecla Schiphorst, and Frédéric Bevilacqua. 2014. Vocalizing Dance Movement for Interactive Sonification of Laban Effort Factors. In Proceedings of the ACM SIGCHI Conference on Designing Interactive Systems (DIS '14). ACM, Vancouver, Canada, 1079-1082.

17. Marco Gillies, Rebecca Fiebrink, Atau Tanaka, Jérémie Garcia, Frederic Bevilacqua, Alexis Heloir, Fabrizio Nunnari, Wendy Mackay, Saleema Amershi, Bongshin Lee, Nicolas d'Alessandro, Joëlle Tilmanne, Todd Kulesza, and Baptiste Caramiaux. 2016. Human-Centred Machine Learning. In Proceedings of the 2016 CHI Conference Extended Abstracts on Human Factors in Computing Systems (CHI'16). ACM, 3558-3565.

18. Kristina Höök, Martin P Jonsson, Anna Ståhl, and Johanna Mercurio. 2016. Somaesthetic Appreciation Design. In Proceedings of the 2016 CHI Conference on Human Factors in Computing Systems (CHI'16). ACM, San Jose, CA, USA, 3131-3142.

19. Caroline Hummels, Kees C. J. Overbeeke, and Sietske Klooster. 2007. Move to get moved: a search for methods, tools and knowledge to design for expressive and rich movement-based interaction. Personal and Ubiquitous Computing 11, 8 (oct 2007), 677-690.

20. David Kirsh. 2013. Embodied cognition and the magical future of interaction design. ACM Transaction on Computer Human Interaction 20, 1 (2013), 1-30.

21. Rudolf Laban and F. C Lawrence. 1974. Effort Economy of Human Movement. Princeton Book.

22. Rudolf Laban and Lisa Ullmann. 1963. Modern educational dance. MacDonald and Evans.

23. Amy LaViers and Magnus Egerstedt. 2012. Style based robotic motion. American Control Conference (ACC) (2012).

24. Wonjun Lee and Richard Shusterman. 2014. Practicing Somaesthetics : Exploring Its Impact on Interactive Product Design Ideation. In Proceedings of the ACM SIGCHI Conference on Designing Interactive Systems (DIS '14). ACM, Vancouver, Canada, 1055-1064. 
25. JA Levy and MP Duke. 2003. Laban Movement Analysis in the study of personality, emotional state and movement style: An exploratory investigation of the veridicality of body language. Individual Differences Research 1, 1 (2003), 39.

26. Lian Loke and George Poonkhin Khut. 2011. Surging Verticality: An Experience of Balance. In Proceedings of the International Conference on Tangible, Embedded, and Embodied Interaction (TEI'11). ACM, 237-240.

27. Lian Loke and Toni Robertson. 2013. Moving and making strange: An embodied approach to movement-based interaction design. Transaction on Computer Human Interaction 20, 1 (2013).

28. Tino Lourens, Roos van Berkel, and Emilia Barakova. 2010. Communicating emotions and mental states to robots in a real time parallel framework using Laban movement analysis. Robotics and Autonomous Systems 58, 12 (2010), 1256-1265.

29. Diego Silang Maranan, Sarah Fdili Alaoui, Thecla Schiphorst, Philippe Pasquier, and Lyn Bartram. 2014. Designing For Movement : Evaluating Computational Models using LMA Effort Qualities. In Proceedings of the 2014 CHI Conference on Human Factors in Computing Systems (CHI'14). ACM, Toronto, Canada.

30. Elena Márquez Segura, Laia Turmo Vidal, Asreen Rostami, and Annika Waern. 2016. Embodied Sketching. In Proceedings of the 2016 CHI Conference on Human Factors in Computing Systems (CHI'16). ACM, San Jose, CA, USA, 6014-6027.

31. M. Masuda, S. Kato, and H. Itoh. 2009. Emotion detection from body motion of human form robot based on laban movement analysis. Springer. 322-334 pages.

32. Helena Mentis and Carolina Johansson. 2013. Seeing Movement Qualities. In Proceedings of the 2013 CHI Conference on Human Factors in Computing Systems (CHI'13). ACM, Paris, France, 3375-3384.

33. M Merleau-Ponty. 1945. Phenomenology of Perception. Editions Gallimard.

34. Jin Moen. 2007. From hand-held to body-worn: embodied experiences of the design and use of a wearable movement-based interaction concept. In Proceedings of the International Conference on Tangible, Embedded, and Embodied Interaction (TEI'07). ACM, 251-258.

35. Marianne Graves Petersen, Ole Sejer Iversen, Peter Gall Krogh, and Martin Ludvigsen. 2004. Aesthetic Interaction: A Pragmatist's Aesthetics of Interactive Systems. In Proceedings of the ACM SIGCHI Conference on Designing Interactive Systems (DIS '04). ACM, Cambridge, MA, USA, 269-276.

36. Mary Pietrowicz, Guy Garnett, Robert McGrath, and John Toenjes. 2010. Multimodal Gestural Interaction in Performance. In Extended Abstracts on Human Factors in Computing Systems (CHI EA '10). ACM, 1-8.
37. Nicolas Rasamimanana, Frédéric Bevilacqua, Norbert Schnell, Emmanuel Fléty, and Bruno Zamborlin. 2011. Modular Musical Objects Towards Embodied Control Of Digital Music Real Time Musical Interactions. In Proceedings of the International Conference on Tangible, Embedded, and Embodied Interaction (TEI '11). ACM, 9-12.

38. TD Sanger. 2007. Bayesian filtering of myoelectric signals. Journal of neurophysiology (2007), 1839-1845.

39. Thecla Schiphorst. 2011. Self-evidence: Applying Somatic Connoisseurship to Experience Design. In $\mathrm{CHI}$ '11 Extended Abstracts on Human Factors in Computing Systems (CHI EA '11). ACM, Vancouver, BC, Canada, $145-160$.

40. Norbert Schnell, Axel Röbel, Diemo Schwarz, Geoffroy Peeters, and Riccardo Borghesi. 2009. Mubu \& friends assembling tools for content based real-time interactive audio processing in max/msp. In Proceedings of International Computer Music Conference. Montreal.

41. Richard Shusterman. 2012. Thinking through the body: Essays in somaesthetics. Cambridge University Press.

42. Karen A Studd and Laura L Cox. 2013. Everybody is a body. Dog Ear Publishing.

43. Gualtierro Volpe. 2003. Computational models of expressive gesture in Computational models of expressive gesture in multimedia systems. Ph.D. Dissertation. InfoMus Lab, Genova.

44. Danielle Wilde, Thecla Schiphorst, and Sietske Klooster. 2011. Move to Design/Design to Move: A Conversation About Designing for the Body. interactions 18, 4 (jul 2011), 22-27.

45. Margaret Wilson. 2009. Dance pedagogy case studies: A grounded theory approach to analyzing qualitative data. Research in Dance Education 10, 1 (2009), 3-16.

46. Liwei Zhao and Norman Badler. 2005. Acquiring and validating motion qualities from live limb gestures. Graphical Models journal 67, 1 (2005), 1-16. 